\title{
Experimental Study on the Dynamic Mechanical Properties of Large-Diameter Mortar and Concrete Subjected to Cyclic Impact
}

\author{
Bi Sun $\mathbb{D}^{1,2}$ Yang Ping, ${ }^{2}$ Zhende Zhu, ${ }^{3}$ Zhijian Jiang, ${ }^{2}$ and Nan Wu $\mathbb{D D}^{4}$ \\ ${ }^{1}$ Shenzhen Key Laboratory of Urban and Civil Engineering for Disaster Prevention and Mitigation, Shenzhen Graduate School, \\ Harbin Institute of Technology, Shenzhen 518055, China \\ ${ }^{2}$ Shenzhen Water Planning and Design Institute Co., Ltd., Shenzhen 518001, China \\ ${ }^{3}$ Key Laboratory of Ministry of Education of Geomechanics and Embankment Engineering, Hohai University, \\ Nanjing 210098, China \\ ${ }^{4}$ Guangzhou University-Tamkang University Joint Research Centre for Engineering Structure Disaster Prevention and Control, \\ Guangzhou University, Guangzhou 510006, China
}

Correspondence should be addressed to Bi Sun; sunbi58@126.com

Received 24 August 2020; Revised 16 November 2020; Accepted 14 December 2020; Published 24 December 2020

Academic Editor: Qing Ma

Copyright (C) 2020 Bi Sun et al. This is an open access article distributed under the Creative Commons Attribution License, which permits unrestricted use, distribution, and reproduction in any medium, provided the original work is properly cited.

\begin{abstract}
Rock bursts are typically accompanied by multiple shocks. In order to explore the dynamic characteristics of filling materials in rock burst roadways, we employ the split Hopkinson pressure bar (SHPB) test to analyze the dynamic mechanical response of mortar and concrete under cyclical impact. The SHPB test results of the large-size specimen indicate the improvement in the waveform shape and the reduction in the wave dispersion via the application of the rubber sheet as the pulse shaper. Under cyclic impact, the peak stress and energy utilization ratio of mortar and concrete specimens were reduced, demonstrating obvious fatigue characteristics. The mortar peak stress and energy utilization ratio were observed to be sensitive to the impact times, while those of concrete were sensitive to impact pressure. The damage evolution of mortar and concrete exhibited very similar trends under the cyclic impact load, whereby the impact pressure had minimal effect on the damage evolution.
\end{abstract}

\section{Introduction}

The development of deep coal mining has increased the complexity of deep coal resource geological conditions [1-5]. In many mining areas in China, controlling rock burst roadway with traditional roadway support methods is difficult, particularly as the rock burst is often accompanied by multiple shocks. Therefore, it is of great significance for engineering practice to study the dynamic characteristics and differences of backfill materials (mortar and concrete) under cyclic impact.

Due to the complexity of underground engineering, the occurrence of rock burst is affected by multiple factors [6]. $\mathrm{Du}$ et al. [7] employed three-dimensional in situ stress measurements in order to investigate rock burst. Zhu et al. [8], Heasley and Tulu [9], and Li et al. [10] each proposed distinct analysis methods for the evaluation of rock burst.
Previous research has effectively determined in situ stress via numerical simulations $[11,12]$.

The split Hopkinson pressure bar (SHPB) test device was first used by Hopkinson to investigate the variations in pressure (bar) with time. This pioneering method has subsequently received much attention due to its ability to study the dynamic mechanical properties of materials [13-15]. Frew et al. [16] determined stress-strain data of rock materials via SHPB technology. The SHPB test device is based on the application of a thin copper sheet on the impact surface of the incident bar to improve the SHPB waveform, thus producing an almost constant strain rate in the specimen. Li et al. [17] carried out repeated impact tests on granite with the SHPB device and revealed the damage caused by each impact to be very low for peak stress values between $60 \%$ and $70 \%$ of the rock static strength. Li et al. [5] examined the degradation process of green sandstone 
subjected to repetitive impact loading by a SHPB apparatus driven with a pendulum hammer. Their test results can improve the understanding of stability evaluations of rock structures subjected to repetitive impact loading. Wang et al. [18] discussed the microproperties of fracture morphology in granite at different temperatures and repeated impacts. Following years of accumulation and development, the complete theory and wide application SHPB technology is currently in place [19-22].

Concrete is a typical heterogeneous material with a large aggregate size and many microdefects. In order to ensure uniformity, the concrete specimen should be of a considerable size; thus, large SHPB devices are required for testing [23]. A key bottleneck of large-diameter SHPB experimental technology is the geometric dispersion caused by the lateral inertia effect when the stress pulse propagates in the compression bar [24, 25]. Wang and Wang [26] indicated that the pulse shaper can eliminate the high frequency oscillation of the incident wave. In particular, the pulse shaper extends the rise time to reduce the lateral inertia effect and is also conducive to constant strain rate loading while also reducing the specimen strain rate. Lee et al. [27] investigated the shaping effect of a brass sheet at varying sizes in a $100 \mathrm{~mm}$ diameter SHPB experiment. Results demonstrated that the smaller the thickness and diameter of the shaper, the longer the rising edge of the incident wave and the smoother the waveform, enhancing the stress uniformity in the specimen. Zhu et al. [28] used the characteristic method to evaluate the stress uniformity of viscoelastic materials in a high strain rate SHPB experiment, revealing the stress uniformity of brittle viscoelastic materials to be affected by the increased incident wave, with negative effects for excessive rise times.

In this paper, cyclic impact compression tests are performed using a SHPB device with mortar and concrete as the research objects. We compare the dynamic mechanics and damage evolution between mortar and concrete under cyclic impact. Our results provide a theoretical reference for research on the stability of roadway filling material under rock bursts.

\section{SHPB Testing Process}

2.1. Specimen Preparation. Ordinary Portland cement and potable laboratory tap water were used for the experiments. Conventional crushed stones with particle sizes between 0.8 and $1.2 \mathrm{~cm}$ and natural river sand were employed as the coarse and fine aggregate, respectively. The concrete was mixed at the proportion of $0.52: 1: 1.67: 2.47$ (water: cement: sand: aggregate) and subsequently set standing for 24 hours. The mold of the specimen was then removed and placed in a constant temperature $\left(20^{\circ} \mathrm{C}\right)$ and humidity (95\%) curing box for 28 days. Following curing, the specimens were drilled and polished to smooth the end face. The mortar specimen was composed of concrete slurry, with preparation and curing methods following those of concrete. The size of both the mortar and concrete specimens was $71 \mathrm{~mm} \times 71 \mathrm{~mm}$ (Figure 1).

2.2. Experimental Principle. The split Hopkinson pressure bar (SHPB) is considered as the most reliable experimental method when investigating the mechanical properties of materials at medium and high strain rates. The SHPB system used in this paper is located at the structural laboratory of Hohai University and operates at the strain rate range $10^{2}-10^{4} \mathrm{~s}^{-1}$. The basic working principle of the SHPB testing device is described as follows. The bullet is pushed by air pressure to impact the incident pressure bar, such that an incident wave $\varepsilon_{I}$ is formed in the bar. Once the incident wave reaches the end of the incident bar, part of it is reflected to form reflected wave $\varepsilon_{R}$. The remaining wave energy passes through the specimen and enters the transmission bar to form transmission wave $\varepsilon_{T}$ (Figure 2).

The strain $\varepsilon_{S}(t)$, strain rate $\dot{\varepsilon}_{S}(t)$, and stress $\sigma_{S}(t)$ of the specimens during the test were calculated as follows:

$$
\begin{aligned}
& \varepsilon_{S}(t)=-\frac{2 C_{0}}{l_{0}} \int_{0}^{t}\left[\varepsilon_{I}(t)+\varepsilon_{R}(t)-\varepsilon_{T}(t)\right] \mathrm{d} t, \\
& \dot{\varepsilon}_{S}(t)=-\frac{2 C_{0}}{l_{0}}\left[\varepsilon_{I}(t)+\varepsilon_{R}(t)-\varepsilon_{T}(t)\right], \\
& \sigma_{S}(t)=\frac{A E_{0}}{2 A_{S}}\left[\varepsilon_{I}(t)+\varepsilon_{R}(t)+\varepsilon_{T}(t)\right],
\end{aligned}
$$

where $C_{0}$ is the propagation velocity of the impact wave in the bar; $l_{0}$ is the specimen thickness; $A$ and $A_{S}$ are the crosssectional area of the bar and the specimen, respectively; $E_{0}$ is the elastic modulus of the bar; and $\varepsilon_{I}(t), \varepsilon_{R}(t)$, and $\varepsilon_{T}(t)$ are the strain signals of the incident, transmission, and reflected waves, respectively.

2.3. Experimental Scheme. Large diameter specimens will result in wave dispersion. In order to improve the initial incident waveform, a pulse shaper is attached to the incident bar [26]. The shaper can "transform" a rectangular pulse into a triangular pulse to lengthen its rising edge [27]. The strain gauge on the transmission bar should be close to the specimen as far as possible, which can also reduce the wave dispersion. Vaseline was applied to the contact surface between the specimen and bar to reduce the end frictional effect. The accuracy of the test data can be improved by applying a filter using the data processing software provided by the SHPB manufacturer. Prior to the experiment, empty bar tests of two brass and butyl rubber sheet pulse shapers were performed, with a shaper diameter and thickness of $20 \mathrm{~mm}$ and $2 \mathrm{~mm}$, respectively.

Figure 3 demonstrates that the dispersion of the waveform is effectively reduced following the filtering. The waveform obtained using the brass shaper is rectangular, while that of the rubber gasket shaper is a half sine wave with a longer rising edge. A half-sine incident waveform can better meet the constant strain rate condition compared to its rectangular counterpart, and thus we selected the rubber sheet pulse shaper for the proceeding experiments.

Multiple impact tests must be performed on the same specimen, and hence the critical impact stress should be determined to ensure that the specimen can withstand multiple impacts. The impact stress at different amplitudes was obtained by controlling the air pressure for cyclic 


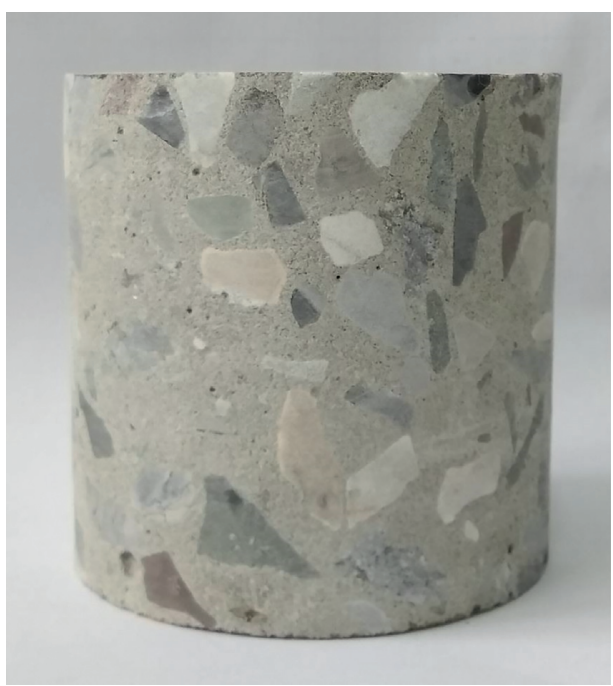

(a)

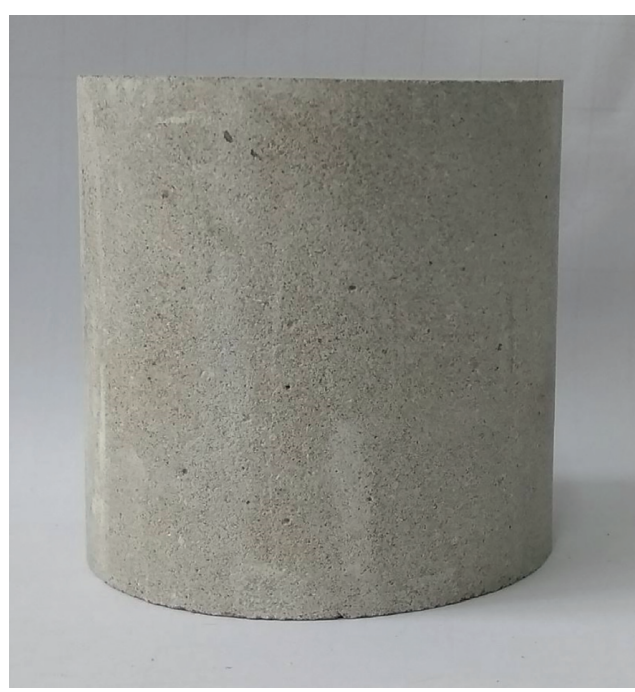

(b)

Figure 1: Specimens used for the SHPB testing. (a) Mortar. (b) Concrete.

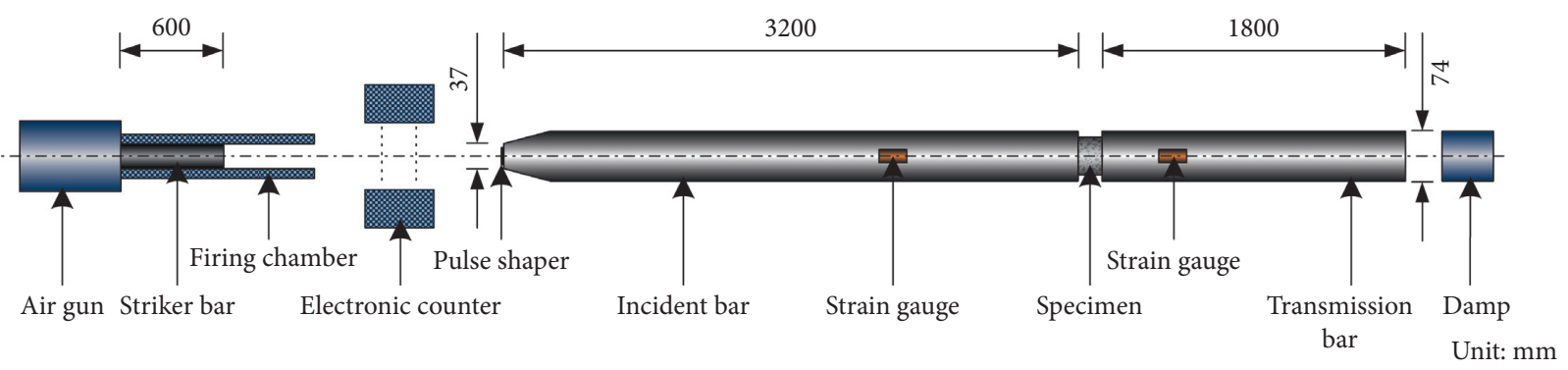

FIgURE 2: Diagram of the split Hopkinson pressure bar test.

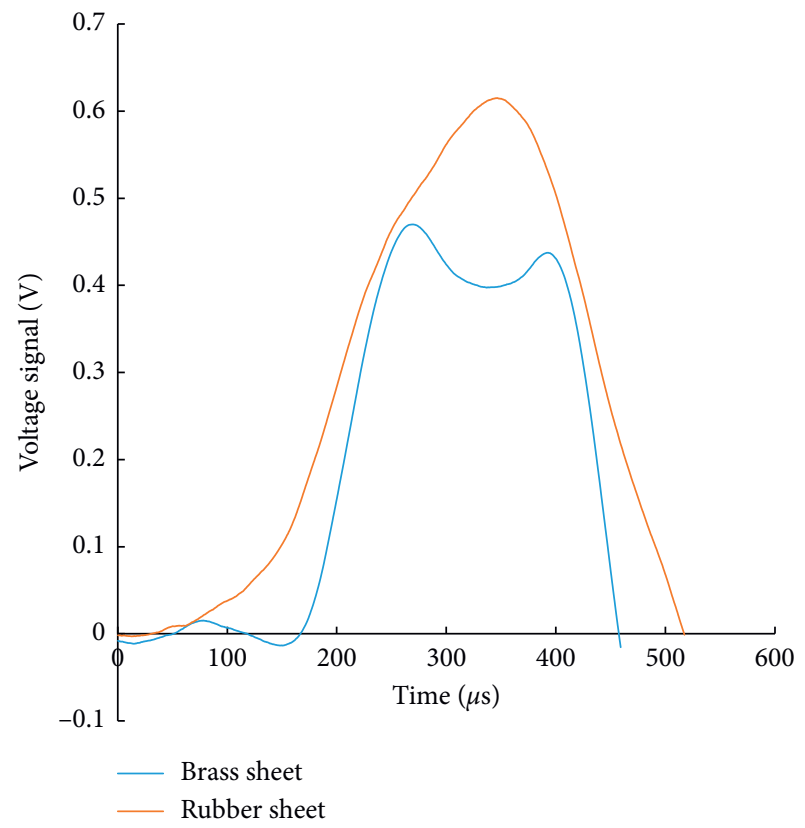

FIgURE 3: Incident waveform of two pulse shapers. impact. According to the characteristics of rock burst and previous experiments, the critical impact pressure value of the mortar and concrete is $0.26-0.28 \mathrm{MPa}$. Therefore, the mortar and concrete were tested with an air pressure of $0.26 \mathrm{MPa}$ and $0.28 \mathrm{MPa}$, respectively.

\section{Experimental Results and Analysis}

3.1. Stress-Strain Curve. Prior to testing the cyclic impact, uniaxial compression tests were performed. The uniaxial compressive strength, strain and elastic modulus of the mortar were determined as $53.07 \mathrm{MPa}, 5.46 \times 10^{-3}$, and 13.10 GPa, respectively (Figure 4). The corresponding values for concrete were $31.23 \mathrm{MPa}, 4.73 \times 10^{-3}$, and $9.97 \mathrm{GPa}$, respectively (Figure 5).

Figure 4 shows that the strength, brittleness, and elastic modulus of the two mortar specimen groups were significantly reduced following the multiple impacts. The strain corresponding to the peak strength of the two groups was not obviously related to the impact times. No obvious differences were observed in the strength reduction between the two air pressure groups. 
Figure 5 shows that, at an air pressure of $0.26 \mathrm{MPa}$, no significant increases were observed in the strength of the first four impact times of the concrete specimen. However, the fifth impact time is associated with an obvious decrease, and the specimen has no bearing capacity. The elastic modulus decreased with the increase in impact time. At an air pressure of $0.28 \mathrm{MPa}$, the strength associated with the second impact was reduced significantly. The elastic modulus exhibited an obvious reduction with increasing impact time.

3.2. Relation between Peak Stress and Number of Cyclic Impacts. Figure 6 presents the relation between the mortar and concrete peak stress and the number of cyclic impacts.

The mortar and concrete peak stress continuously decreased with the increasing impact time, indicating the continuous deterioration of the bearing capacity. The peak stress of mortar specimens varies with the impact pressure (Figure 6(a)), while the slopes of the two curves are almost equal. As the number of cyclic impacts increased, the peak stress reduction rate of mortar stabilized. Following the fourth impact time, the peak stress of specimen CC-1 decreased from 120.26 MPa to $22.37 \mathrm{MPa}$ (81.40\%), while the peak stress of specimen CC-2 decreased from 142.40 $\mathrm{MPa}$ to $58.15 \mathrm{MPa}$ (59.16\%). Despite its low impact pressure, specimen CC-1 exhibited a marked decrease in peak stress.

Specimens $\mathrm{HC}-1$ and $\mathrm{HC}-2$ had air pressures of $0.26 \mathrm{MPa}$ and $0.28 \mathrm{MPa}$, respectively (Figure 6(b)), with the slope of HC-2 curve double that of HC-1. More specifically, a rise in the impact number increased the impact pressure, which consequently resulted in a steeper reduction of the concrete peak stress. After repeated impacts, the peak stress of specimen HC-1 decreased from $74.92 \mathrm{MPa}$ to $40.93 \mathrm{MPa}(45.37 \%)$, while that of specimen HC-2 decreased from 87.61 MPa to $46.54 \mathrm{MPa}(46.88 \%)$. The two specimens exhibit an almost equal reduction in peak stress.

The fitting degree of the mortar specimen is higher than that of concrete. Although the homogeneity of the concrete specimens could be ensured by the use of large-size specimens, the dispersion of test data was still relatively large. Under the same impact pressure, the initial peak stress of the mortar was greater than that of concrete. The absolute value of the mortar slope was also larger than that of concrete, indicating the sensitivity of the mortar peak stress to the impact time. Moreover, the two mortar curves are parallel, while those of concrete intersect. The results demonstrate the greater sensitivity of the peak stress to the impact pressure.

3.3. Relation between the Energy Utilization Ratio and Number of Cyclic Impacts. The energy carried by the incident and transmission waves and energy utilization rate between loading and unloading were determined as follows:

$$
\begin{aligned}
W_{I} & =E_{0} A C_{0} \int_{0}^{t} \varepsilon_{I}^{2} \mathrm{~d} t, \\
W_{T} & =E_{0} A C_{0} \int_{0}^{t} \varepsilon_{T}^{2} \mathrm{~d} t, \\
\eta & =\frac{W_{T}}{W_{I}} \cdot 100 \%,
\end{aligned}
$$

where $W_{I}, W_{T}$, and $\eta$ are the incident energy, transmission energy, and energy utilization ratio, respectively. Figure 7 depicts the relation between the energy utilization ratio of mortar and concrete and number of cyclic impacts.

The curves in Figures 6 and 7 exhibit similar trends. The energy utilization ratio of both the mortar and concrete specimens decreased as the number of impact cycles increased. The energy utilization ratio of the mortar specimen varies with impact pressure (Figure 7(a)), while the slopes of the two curves are almost equal. In particular, under the two impact pressures, the rate of reduction of the mortar peak stress is almost equal to the increase in cyclic impact times. Following the fourth impact time, the energy utilization ratio of specimen CC-1 decreased from $7.89 \%$ to $0.24 \%$ (96.96\%), while that of specimen CC-2 decreased from $10.50 \%$ to $1.64 \%(84.38 \%)$. The energy utilization ratio of specimens CC-1 and CC-2 decreased greatly following the repeated impacts. This indicates close to complete breakage of the specimen.

The slope of the specimen $\mathrm{HC}-2$ curve is 2.64 times that of HC-1 (Figure 7(b)), revealing that the higher the impact number, the greater the impact pressure and the steeper the reduction in the energy utilization ratio of the concrete samples. After repeated impacts, the energy utilization ratio of specimen HC-1 decreased from $4.71 \%$ to $0.74 \%(84.29 \%)$, while that of specimen HC-2 decreased from $7.50 \%$ to $0.97 \%$ (87.07\%). The reduction ranges are almost equal. Moreover, the fitting degree of the mortar sample is greater than that of concrete.

Under the same impact pressure, the initial energy utilization ratio of mortar was higher than that of concrete. The absolute value of the mortar slope is larger than that of concrete, indicating that the energy utilization ratio of mortar is more sensitive to the impact times. Furthermore, the two mortar curves are parallel, while those of concrete intersect. This demonstrates that the energy utilization ratio of concrete is more sensitive to the impact pressure.

3.4. Relation between Damage Degree and Number of Cyclic Impacts. The load-unload response ratio (LURR) was initially proposed by Yin Xiangchu [29], who extracted the damage degree of the target medium based on the concept of "the change of physical quantity." The LURR is used to quantify the damage of the medium. The common axial stress is employed as the LURR load variable, and the corresponding strain is used as the response variable. The LURR $(Y)$ is defined as follows: 


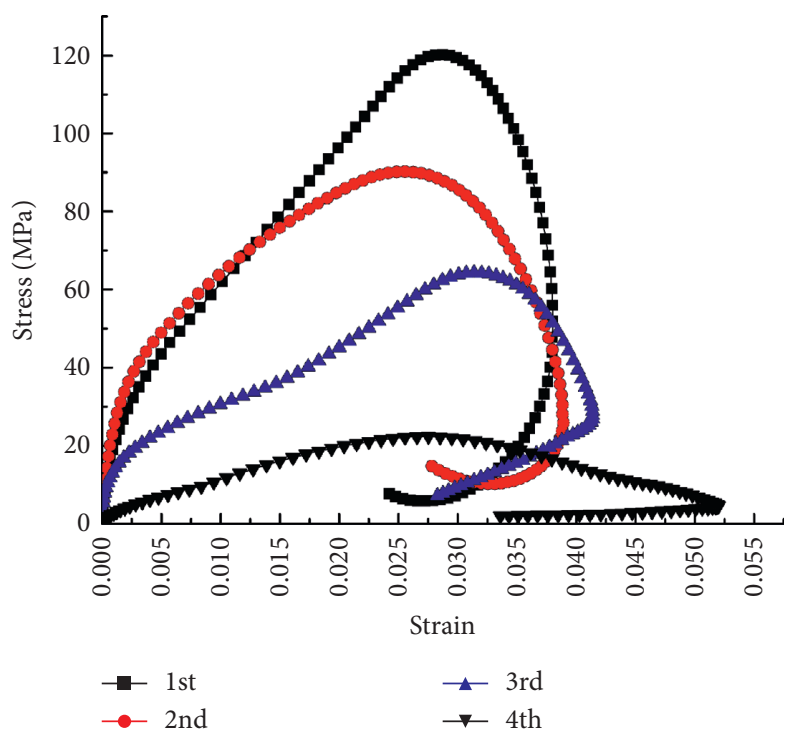

(a)

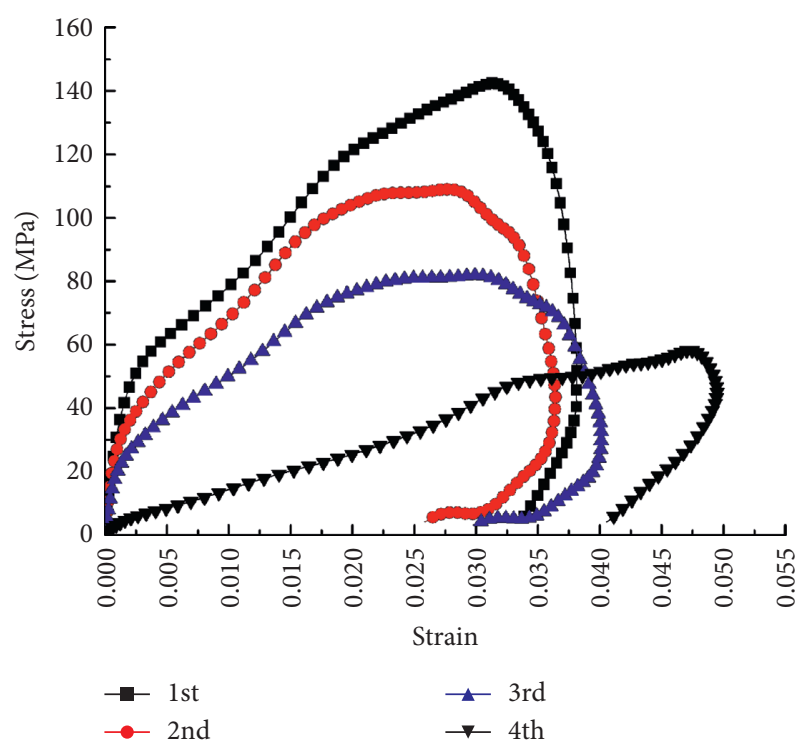

(b)

Figure 4: Cyclic impact stress-strain curves of mortar under different air pressures. (a) CC-1 (air pressure of 0.26 MPa); (b) CC-2 (air pressure of $0.28 \mathrm{MPa}$ ).

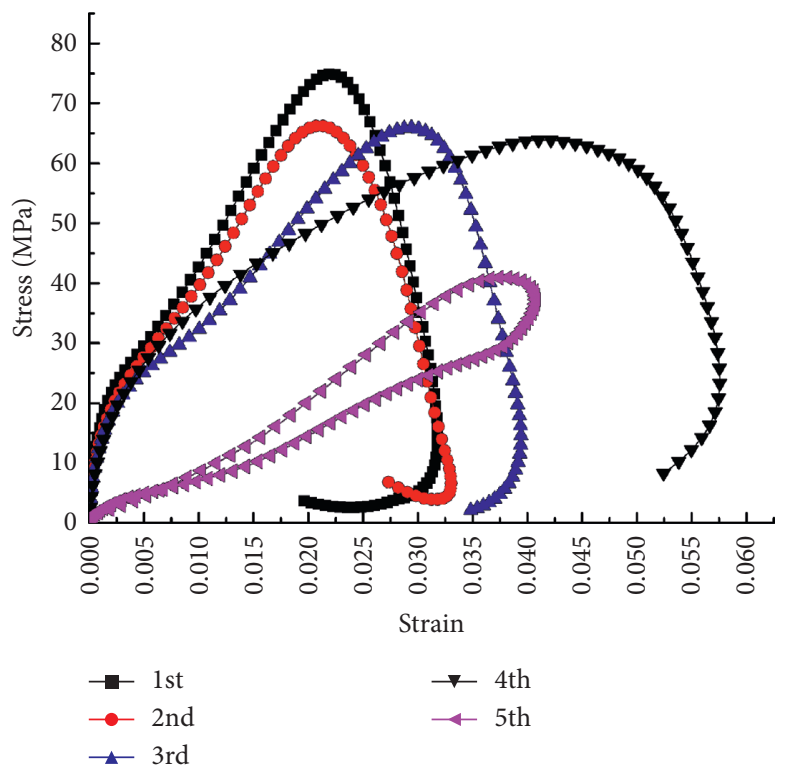

(a)

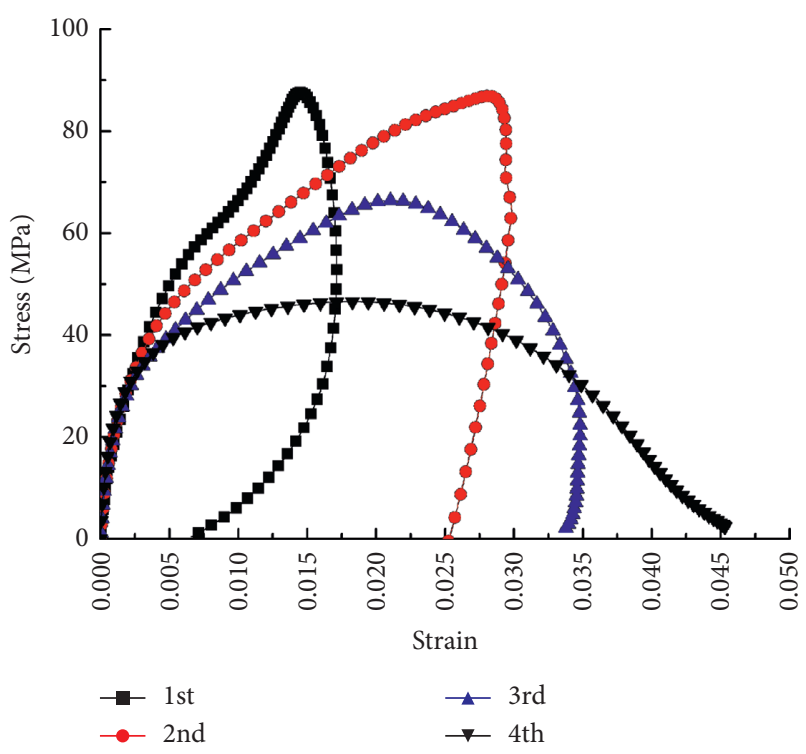

(b)

Figure 5: Cyclic impact stress-strain curves of concrete under different air pressures. (a) HC-1 (air pressure of 0.26 MPa); (b) HC-2 (air pressure of $0.28 \mathrm{MPa}$ ).

$$
Y=\frac{X_{+}}{X_{-}}
$$

where $X_{+}$and $X_{-}$are the response rate under loading and unloading conditions, respectively, and are defined as follows:

$$
X=\lim _{\Delta P \longrightarrow 0} \frac{\Delta R}{\Delta P}
$$

where $\Delta P$ and $\Delta R$ denote the increments of load $P$ and response $R$, respectively.

For the elastic phase, the response rate $X_{+}=X_{-}$, and thus, $Y=1$. However, $X_{+}>X_{-}$occurs when the load exceeds the elastic limit, and thus, $Y>1$. The more severe the damage of the material, the larger the $Y$ value. When the system approaches instability, $Y \longrightarrow \infty$.

Based on the assumption that the damage at the fracture limit obeys the Weibull distribution on the mesoscale 


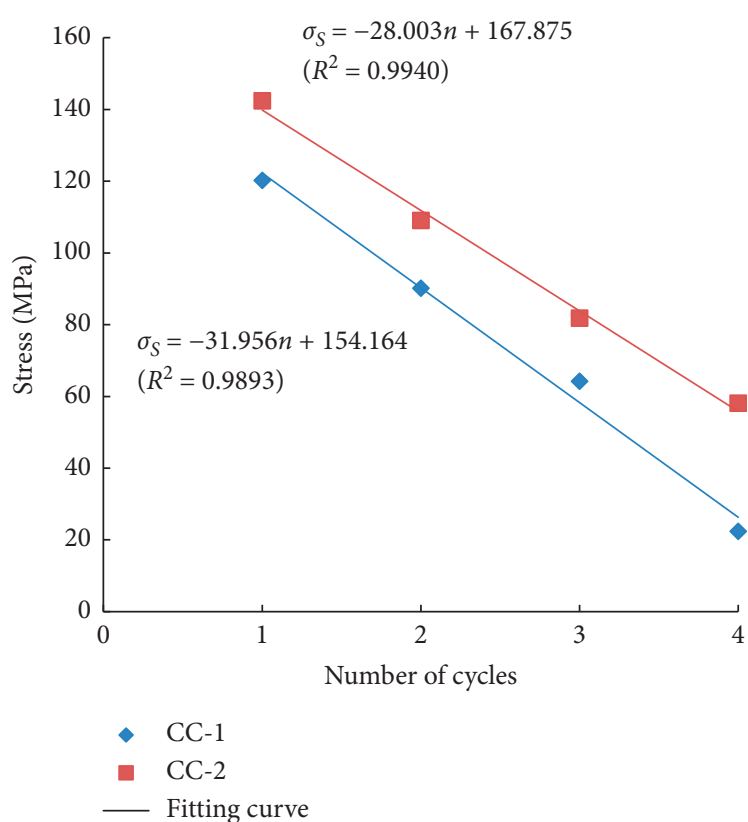

(a)

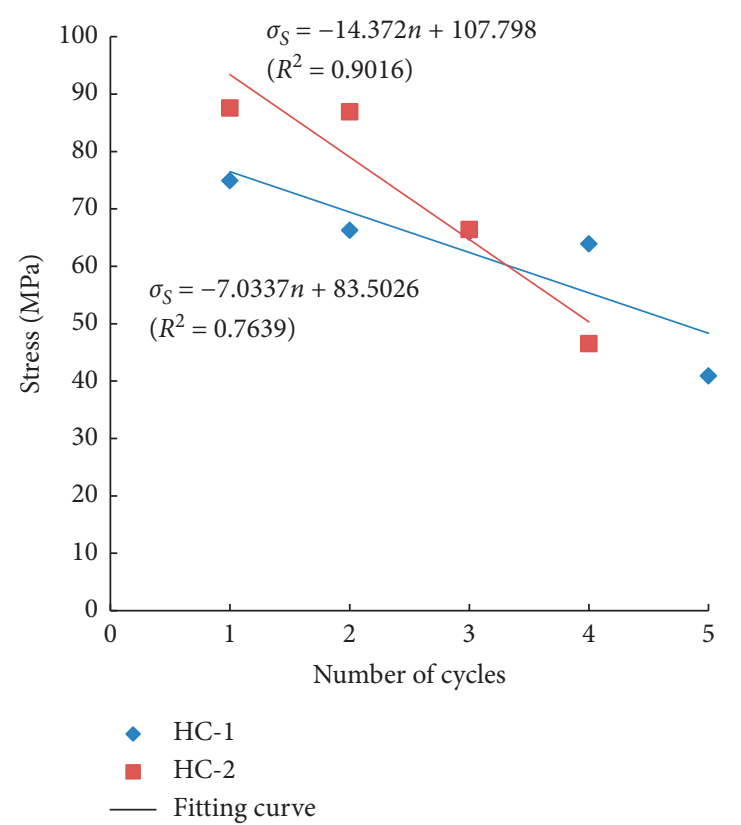

(b)

FIgURE 6: Relation between peak stress and the number of cyclic impacts. (a) Mortar. (b) Concrete.

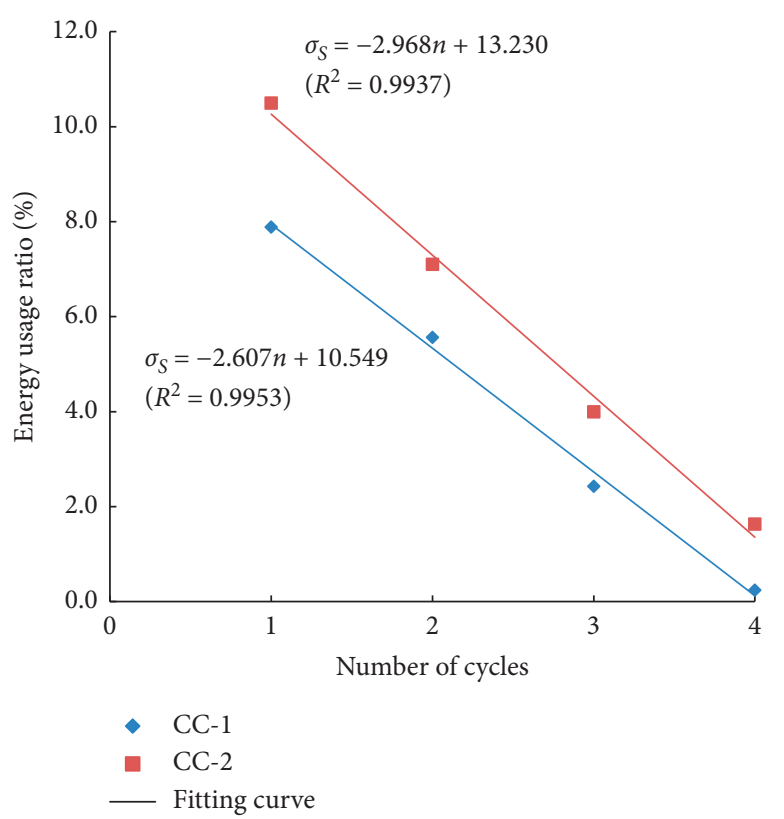

(a)

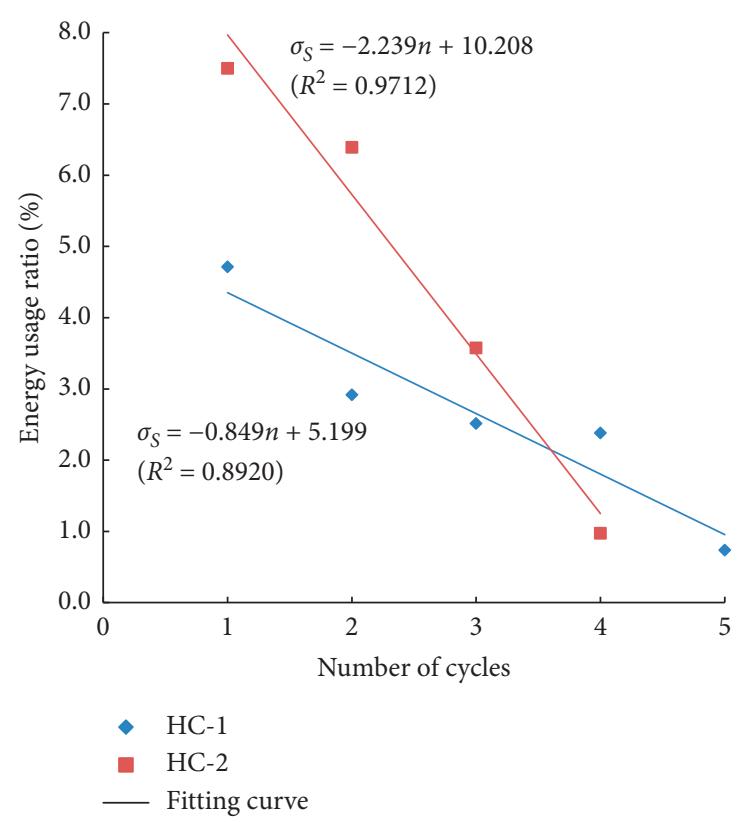

(b)

FIGURE 7: Relation between the energy utilization ratio and number of cyclic impacts. (a) Mortar. (b) Concrete.

[30-32], Zhang et al. [33, 34] established a relationship between $Y$ and the damage variable $D$ as follows:

$$
D=1-\exp \left(\frac{1-Y}{m Y}\right)
$$

where $m$ is the Weibull index.
The loading and unloading section secant slopes of the stress-strain curve were used to calculate the LURR, allowing for the damage degree of the specimen following each impact to be determined (Figure 8).

The first impact was the most damaging for both the mortar and concrete samples. In particular, damage to the mortar specimens CC-1 and CC-2 was 0.73 and 0.70 , 


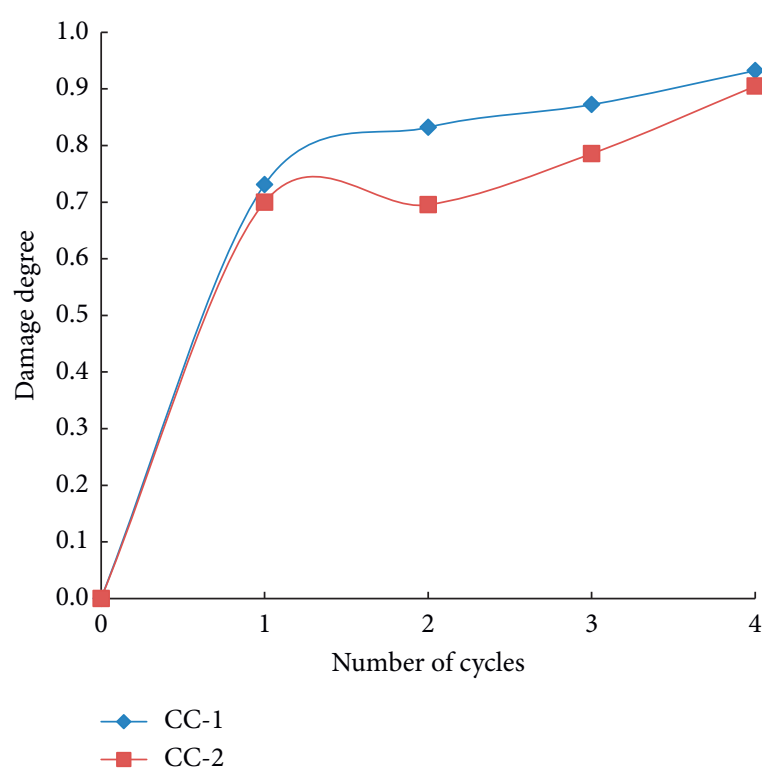

(a)

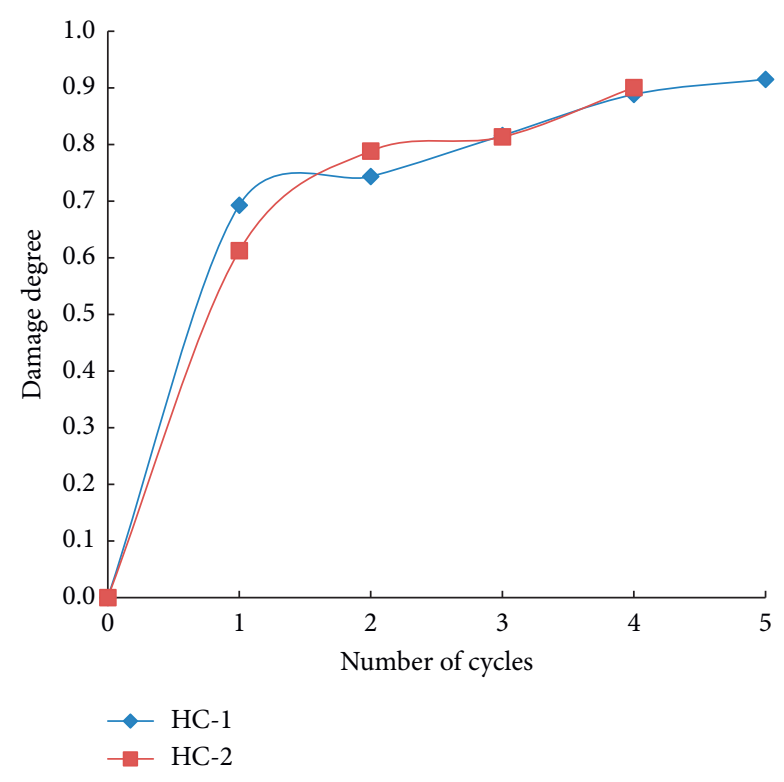

(b)

FIGURE 8: Relation between damage degree and number of impact cycles $t$. (a) Mortar. (b) Concrete.

accounting for $78.42 \%$ and $77.35 \%$ of the maximum damage, respectively (Figure $8(\mathrm{a})$ ). In the subsequent cyclic impacts, the increase in damage of specimen CC-1 was relatively minimal, with a damage increment less than 0.11 . When the damage reached 0.93 , the load was unbearable. The damage increment of specimen CC-2 approached 0 at the second impact, while at the third and fourth impact, the damage increment of the specimen increased rapidly. When the damage reached 0.90 , the specimen could not bear the load. The maximum damage increment of the last three impacts was 0.12 .

The damage of concrete specimens $\mathrm{HC}-1$ and $\mathrm{HC}-2$ at the first impact was observed as 0.78 and 0.70 , accounting for $77.98 \%$ and $68.00 \%$ of the maximum damage, respectively (Figure 8(b)). During the subsequent cyclic impacts, the damage accumulation of the two specimens exhibited slight fluctuations. The maximum damage increment of $\mathrm{HC}-1$ was observed as 0.072 , while that of $\mathrm{HC}-2$ was 0.018 . The two specimens were unable to bear their loads once the damage reached 0.92 and 0.90 , respectively.

The results demonstrate the strong similarity between the damage evolution trends of the mortar and concrete specimens under cyclic impact loads. At the initial impact, the damage was greater than 0.7 . When the damage exceeded 0.9 , the specimen no longer had a bearing capacity. Thus, when the damage degree of the first impact was greater than 0.7, the effects of the impact times and pressure on the mortar and concrete damage evolution were relatively small.

\section{Conclusion}

The current paper is based on the assumption that rock bursts are typically followed by continuous multiple shocks. Filling materials, mortar and concrete, were selected as the research objects to investigate the mechanical properties of mortar and concrete under cyclic impacts. The following conclusions were determined:

(1) The SHPB testing of large-size specimens revealed the improvement of the waveform shape and the reduction in the wave dispersion via the application of a rubber sheet as the pulse shaper. Following repeated impacts, the peak stress of the mortar and concrete specimens was reduced. This indicates obvious fatigue characteristics.

(2) Although the large-size concrete specimens could maintain a certain level of homogeneity, the dispersion was relatively large. The peak stress of mortar was sensitive to the impact time, while that of concrete was sensitive to impact pressure.

(3) As the number of cycles increased, the energy utilization ratio of the mortar and concrete specimens decreased. Under the same impact pressure, the initial energy utilization ratio of mortar was higher than that of concrete. Furthermore, the energy utilization ratio of mortar was sensitive to the impact time, while that of concrete was more sensitive to the impact pressure.

(4) Trends in the damage evolution of mortar and concrete were in strong agreement under the cyclic impact load. The impact pressure had a minimal effect on the damage evolution.

\section{Data Availability}

The experimental data used to support the findings of this study are available from the corresponding author upon request. 


\section{Conflicts of Interest}

The authors declare that they have no conflicts of interest.

\section{Acknowledgments}

This research was funded by the National Natural Science Foundation of China (Grant nos. 41831278, 51579081, and 51709184) and Central Public-Interest Scientific Institution Basal Research Fund (Grant no. Y118008).

\section{References}

[1] Y. Jiang, Y. Zhao, H. Wang et al., "A review of mechanism and prevention technologies of coal bumps in China," Journal of Rock Mechanics and Geotechnical Engineering, vol. 9, no. 1, pp. 180-194, 2017.

[2] X. Pang, Y. Jiang, Y. Zhao, and J. Zhu, "Study on risk analysis and control technology of coal bump," Procedia Environmental Sciences, vol. 12, pp. 831-836, 2012.

[3] S. Qin, J. J. Jiao, C. A. Tang et al., "Instability leading to coal bumps and nonlinear evolutionary mechanisms for a coalpillar-and-roof system," International Journal of Solids and Structures, vol. 43, no. 25-26, pp. 7407-7423, 2006.

[4] Y. L. Tan, X. S. Liu, B. Shen et al., "New approaches to testing and evaluating the impact capability of coal seam with hard roof and/or floor in coal mines," Geomechanics and Engineering, vol. 14, no. 4, pp. 367-376, 2018.

[5] S. H. Li, W. C. Zhu, L. L. Niu et al., "Dynamic characteristics of green sandstone subjected to repetitive impact loading: phenomena and mechanisms," Rock Mechanics and Rock Engineering, vol. 51, no. 6, pp. 1921-1936, 2018.

[6] Q. Qian and X. Zhou, "Failure behaviors and rock deformation during excavation of underground cavern group for jinping I hydropower station," Rock Mechanics and Rock Engineering, vol. 51, no. 8, pp. 2639-2651, 2018.

[7] K. Du, M. Tao, X. Li et al., "Experimental study of slabbing and rockburst induced by true-triaxial unloading and local dynamic disturbance," Rock Mechanics and Rock Engineering, vol. 49, no. 9, pp. 3437-3453, 2016.

[8] G. Zhu, L. Dou, A. Cao et al., "Assessment and analysis of strata movement with special reference to rock burst mechanism in island longwall panel," Journal of Central South University, vol. 24, no. 12, pp. 2951-2960, 2017.

[9] K. A. Heasley and I. B. Tulu, "Using LaModel to analyze coal bumps," International Journal of Mining Science and Technology, vol. 28, no. 5, pp. 729-737, 2018.

[10] Z. Li, Y. Xue, S. Li et al., "Rock burst risk assessment in deepburied underground caverns: a novel analysis method," Arabian Journal of Geosciences, vol. 13, no. 11, 2020.

[11] L. Weng, L. Huang, A. Taheri, and X. Li, "Rockburst characteristics and numerical simulation based on a strain energy density index: a case study of a roadway in Linglong gold mine, China," Tunnelling and Underground Space Technology, vol. 69, pp. 223-232, 2017.

[12] Q. Jiang, X. Feng, T. Xiang et al., "Rockburst characteristics and numerical simulation based on a new energy index: a case study of a tunnel at 2,500 $\mathrm{m}$ depth," Bulletin of Engineering Geology and the Environment, vol. 69, no. 3, pp. 381-388, 2010.

[13] B. Hopkinson, "A method of measuring the pressure produced in the detonation of high explosives or by the impact of bullets," Philosophical Transactions of the Royal Society of London. Series A, Containing Papers of a Mathematical or Physical Character, vol. 89, pp. 411-413, 1914.
[14] G. I. Taylor, "The testing of materials at high rates of loading," Journal of the Institution of Civil Engineers, vol. 26, no. 8, pp. 486-519, 1946.

[15] H. Kolsky, "An investigation of the mechanical properties of materials at very high rates of loading," Proceedings of the Physical Society. Section B, vol. 62, no. 11, pp. 676-700, 1949.

[16] D. J. Frew, M. J. Forrestal, and W. Chen, "A split Hopkinson pressure bar technique to determine compressive stress-strain data for rock materials," Experimental Mechanics, vol. 1, no. 41, pp. 40-46, 2001.

[17] X. B. Li, T. S. Lok, and J. Zhao, "Dynamic characteristics of granite subjected to intermediate loading rate," Rock Mechanics and Rock Engineering, vol. 38, no. 1, pp. 21-39, 2005.

[18] P. Wang, P. Wang, T. Yin et al., "Dynamic properties of thermally treated granite subjected to cyclic impact loading," Rock Mechanics and Rock Engineering, vol. 52, no. 4, pp. 991-1010, 2019.

[19] J. Zhou and L. Ge, "Effect of strain rate and water-to-cement ratio on compressive mechanical behavior of cement mortar," Journal of Central South University, vol. 22, no. 3, pp. 1087-1095, 2015.

[20] H.-b. Du, F. Dai, Y. Xu, Y. Liu, and H.-n. Xu, "Numerical investigation on the dynamic strength and failure behavior of rocks under hydrostatic confinement in SHPB testing," International Journal of Rock Mechanics and Mining Sciences, vol. 108, pp. 43-57, 2018.

[21] N. Wu, J. Fu, Z. Zhu, and B. Sun, "Experimental study on the dynamic behavior of the Brazilian disc sample of rock material," International Journal of Rock Mechanics and Mining Sciences, vol. 130, p. 104326, 2020.

[22] N. Wu, Z. Zhu, C. Zhang et al., "Dynamic behavior of rock joint under different impact loads," KSCE Journal of Civil Engineering, vol. 23, no. 2, pp. 541-548, 2019.

[23] X. T. Wu, S. S. Hu, B. Y. Yang et al., "An improvement in measuring the dynamic properties of concrete material by the traditional SHPB technique," Journal of Hefei University of Technology, vol. 1, pp. 63-66, 2004.

[24] C. Chree, "The equations of an isotropic elastic solid in polar and cylindrical Co-ordinates their solution and application," Transactions of the Cambridge Philosophical Society, vol. 14, pp. 250-369, 1889.

[25] L. Pochhammer, "Ueber die fortpflanzungsgeschwindigkeiten kleiner schwingungen in einem unbegrenzten isotropen kreiscylinder," Journal für die reine und angewandte MathematikPY, vol. 81, pp. 324-336, 1876.

[26] Y. G. Wang and L. L. Wang, "Stress wave dispersion in largediameter SHPB and its manifold manifestations," Journal of Beijing Institute of Technology, vol. 3, pp. 247-253, 2004.

[27] O. S. Lee, S. H. Kim, and Y. H. Han, "Thickness effect of pulse shaper on dynamic stress equilibrium and dynamic deformation behavior in the polycarbonate using SHPB technique," Journal of Experimental Mechanics, vol. 21, no. 1, pp. 51-60, 2006.

[28] J. Zhu, S. Hu, and L. Wang, "An analysis of stress uniformity for concrete-like specimens during SHPB tests," International Journal of Impact Engineering, vol. 36, no. 1, pp. 61-72, 2009.

[29] X. Yin, L. Zhang, H. Zhang et al., "LUR's twenty years and its perspective," Pure and Applied Geophysics, vol. 163, no. 11-12, pp. 2317-2341, 2006.

[30] X. H. Xu, S. P. Ma, M. F. Xia et al., "Damage evaluation and damage localization of rock," Theoretical and Applied Fracture Mechanics, vol. 42, no. 2, pp. 131-138, 2004.

[31] Y.-J. Wei, M.-F. Xia, F.-J. Ke, X.-C. Yin, and Y.-L. Bai, "Evolution-induced catastrophe and its predictability," $\mathrm{Mi}$ croscopic and Macroscopic Simulation: Towards Predictive 
Modelling of the Earthquake Process, vol. 157, no. 11-12, pp. 1945-1957, 2000.

[32] W. Weibull, "A statistical distribution function of wide applicability," Transactions of the ASME, Journal of Applied Mechanics, vol. 2, no. 18, pp. 293-297, 1951.

[33] L. Zhang, H. Yu, and X. Yin, "Failure potential evaluation in engineering experiments using load/unload response ratio method," Pure and Applied Geophysics, vol. 170, no. 1-2, pp. 237-245, 2013.

[34] B. Sun, Z. Zhu, C. Shi, and Z. Luo, "Dynamic mechanical behavior and fatigue damage evolution of sandstone under cyclic loading," International Journal of Rock Mechanics and Mining Sciences, vol. 94, pp. 82-89, 2017. 